\title{
Rejuvenating Effects of Facial Hydrofilling using Restylane Vital
}

\author{
Bong Moo Lee ${ }^{1}$, Dong Gil Han ${ }^{1}$, Won Seok Choi ${ }^{2}$ \\ ${ }^{1}$ Department of Plastic and Reconstructive Surgery, Catholic University of Daegu School of Medicine, Daegu; ${ }^{2}$ V- Plastic Surgery Group, \\ Daegu, Korea
}

Background Morphological changes that accompany aging, such as wrinkles and skin laxity, are particularly prominent on facial skin. Recently, facial rejuvenation using the hydrofilling effect of hyaluronic acid (HA) filler has been employed for improvement of skin texture. In this study, we studied rejuvenating effects of stabilized HA (Restylane Vital) through direct intradermal injections.

Methods A total of 30 female patients underwent a series of procedures on face, including three sessions at intervals of four weeks. A total of $2 \mathrm{~mL}$ of Restylane Vital was injected along the whole face using an automatic injector. Improvement of skin surface roughness, elasticity, brightness, moisture, and fine wrinkles was evaluated. Patient satisfaction was evaluated, and pictures of patients were taken at each visit and 6 months after last treatment session. Scoring for each patient was performed by three doctors according in five subjects. Moisture, oil and elasticity were measured before the procedure and before the last treatment in 10 patients.

Results The majority of patients (77\%) were satisfied with the therapeutic outcomes. Approximately $66 \%$ of patients responded that the effects of this procedure persisted for longer than four months, and the majority of patients (77\%) wanted to undergo this procedure again and would recommend this procedure to acquaintances. Regarding doctors' evaluation, scores for improvement of skin surface roughness, elasticity, and brightness were significantly higher than those for improvement of moisture and fine wrinkle.

Conclusions Intradermal injection of HA can have a rejuvenating effect on dry and tired facial skin, especially in improvement of skin surface roughness.

Keywords Hyaluronic acid / Injection, intradermal / Rejuvenation
Correspondence: Dong Gil Han Department of Plastic and Reconstructive Surgery, Catholic University of Daegu School of Medicine, 33 Duryugongwon-ro 17-gil, Nam-gu, Daegu 705-718, Korea

Tel:+82-53-650-4580

Fax: +82-53-650-4584

E-mail: dghan1001@cu.ac.kr

No potential conflict of interest relevant to this article was reported.

Received: 8 May 2014 • Revised: 20 May 2014 • Accepted: 21 May 2014

pISSN: 2234-6163 • elSSN: 2234-6171 • http://dx.doi.org/10.5999/aps.2015.42.3.282 • Arch Plast Surg 2015;42:282-287

\section{INTRODUCTION}

With advancing age, the concentration of collagen with elastic fibers and repair process of connective tissue are decreased, especially in hydration of skin. Because of its hydrophilic nature, hyaluronic acid (HA) serves a biological role in creation of volume and lubrication of intracellular structure. In addition, con- centration of HA in skin decreases with aging. As a result, elasticity and ability to hold water in skin are decreased, leading to decreased volume of dermis, and increased tendency of wrinkle and skin laxity formation on particularly facial skin; these factors can have a negative psychosocial impact. Due to little burden and rapid recovery, a number of patients have recently preferred minimally or non-invasive procedures such as filler injection. 
HA filler has been used for improvement of facial wrinkles. Recently, by injection of HA filler into intradermal layers, people have been interested in improvement of skin texture, not improvement of deep wrinkles and volume deficiency, which was an issue in the past. To date, only a few studies have reported on the facial rejuvenating effects of HA filler. In this study, the author described a skin texture improvement using hydrofilling of Restylane Vital.

\section{METHODS}

This study was a single-center, prospective study. A total of 30 female patients with dry and tired skin on face were enrolled in the study. The age range of patients was from 27 to 60 years. All patients provided written, informed consent. Patients were excluded if they were pregnant or breast feeding, if they had active skin disease (such as infection, eczema, various dermatitis, etc.), or if they had a known hypersensitivity to HA, autoimmune disease, or skin cancer. In addition, patients who had been treated with other skin rejuvenation procedures, such as peeling, botulinum toxin, other filler, or laser therapy and had undergone facial surgery within the previous 12 months were excluded. 5\% lidocaine ointment was applied to the entire facial area at least 30 minutes before treatment. After wiping off the lidocaine with gauze, acetone was used to completely wipe it off. Using an automatic intradermal injector, $2 \mathrm{~mL}$ of Restylane Vital (Q-Med, Uppsala, Sweden) mixed with $3 \mathrm{~mL}$ of normal saline and $1 \mathrm{~mL}$ of $2 \%$ lidocaine was injected into the whole face per treatment session (approximately 100 point $\times 0.02 \mathrm{~mL}$ injection of Restylane Vital per session). No additional procedures were performed simultaneously. Multiple injections were administered in intradermal layer using AQUAGENT (M'CURE, Wonju, Korea). We use AQUAGENT for precise injection to intradermal layer in small amount of HA filler. Eligible patients received injection of HA into whole face from forehead to chin in three sessions at intervals of four weeks. In order to minimize the risk of complication, such as hematoma, the injection site was gently compressed, and cooled with an ice pack for several minutes after procedures.

A photograph of the patient's face was taken before each treatment session and 6 months after last treatment session. In order to standardize the quality of photographs, standard lightening and views were used with a Nikon D80 SLR digital camera with a flash unit installed within the camera. All images were taken by the same photographer.

Patients were also asked about their thoughts regarding aesthetic satisfaction using questionnaire survey after 6 months of last procedure. The grade of satisfaction was checked from point
1 to 5 (point 1, very unsatisfied; point 2, unsatisfied; point 3, ok; point 4, satisfied; point 5, very satisfied). Four questions were "Do you think this procedure was effective?", "How long last the effect of this procedure?", "Would you like to undergo this procedure again?", and "Would you be willing to recommend this procedure to acquaintances (friends)?”

Three plastic surgeons performed scoring according to a 5-point scale for each patient in five subjects (Improvement of skin surface roughness, fine wrinkles, moisture, brightness and elasticity). The collected data was statistically measured by mean value and standard deviation (SD). Statistical significance testing used one sample t-test and one way ANOVA, using IBM SPSS ver. 19.0 (IBM Co., Armonk, NY, USA). The Scheffe method was used in analysis of the results of multiple comparisons. P-value of less than 0.05 was considered to indicate statistical significance.

Moisture, oil, and elasticity meter (Triple sense, Moritex Co., Saitama, Japan) were measured before performance of the first procedure and the last procedure. In the dressing room condition, constant temperature and humidity were maintained for accurate measurement of skin condition. After cleansing of the face, oil, moisture, and elasticity meter were measured. In order to reduce measurement errors, patients' cheeks were measured on both sides two times, and the average value was used. The collected data were measured statistically by mean value and SD. Statistical significance testing used paired samples t-test, using IBM SPSS ver. 19.0.

\section{RESULTS}

Of 30 patients, all patients completed the treatment session. The mean age of patients was 46.4 years. No serious adverse events occurred during the course of treatment. Three patients experienced temporary, mild erythema after treatment, which subsided one week later and healed without any sequelae. Two patients had transient, mild hematomas after treatment.

Patient satisfaction was evaluated using four questions.

Question 1: Do you think this procedure was effective? Approximately 28 patients (94\%) scored above 3 points, and 23 patients (77\%) scored above 4 points. The majority (77\%) of patients rated the therapeutic success as "very satisfied" or "satisfied". Only two patient stated that outcome was "unsatisfied" (Fig. 1).

Question 2: How long last the effect of this procedure? Twenty patients $(66 \%)$ responded that the effects of this procedure persisted for longer than 4 months (Fig. 2).

Question 3: Do you want to undergo this procedure again? Twenty-two patients (77\%) answered "Yes". 
Question 4: Would you be willing to recommend this procedure to acquaintances (friends)? Twenty-two patients (77\%) answered "yes".

As a result of comparison of five subjects regarding evaluation of doctors, improvement of five subjects was changed significantly $(\mathrm{P}<0.05)$. And improvement of brightness showed the highest score and follow by improvement of skin surface roughness and elasticity (Table 1 ). There were no significant differences by evaluator, thus the doctors were objective in score assignment (Table 2). Scores for improvement scores of brightness, skin surface roughness and elasticity were significantly higher than those for improvement of fine wrinkle and moisture (Table 3). The evaluations showed statistically significant changes in moisture, oil and elasticity improvement $(\mathrm{P}<0.05)$.

\section{Fig. 1. Result of question 1 in four questions}

The result for satisfaction in 30 patients is shown with the question "Do you think this procedure was effective?" Twenty eight patients (94\%) scored above 3 points, and 23 patients (77\%) scored above 4 points.

Question 1: Do you think this procedure was effective? (1-5 point)

$\square 1 \square 2 \square 3 \square 4 \square 5$ (point) Point 1: very unsatisfied

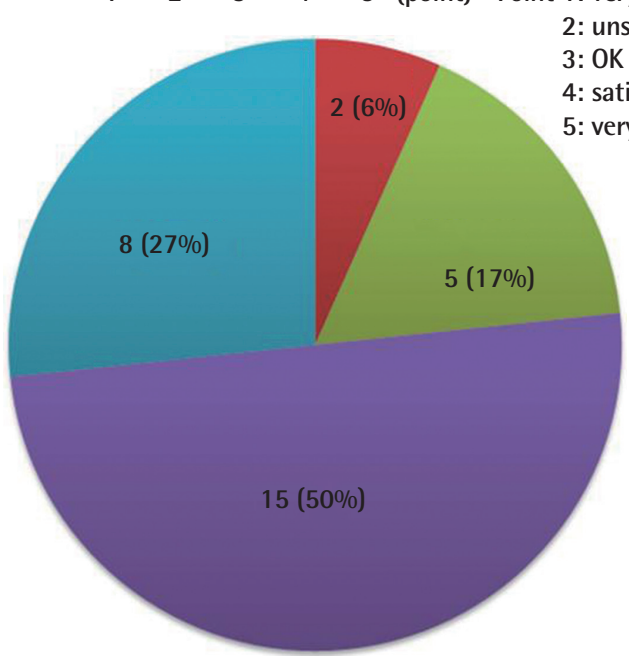

No. of patients $(\%)$
Elasticity was the most improved and followed by moisture and oil (Table 4).

Case 1: a 35-year-old female patient was injected at whole face

\section{Fig. 2. Result of question 2 in four questions}

The result for satisfaction in 30 patients is shown with the question "How long last the effect of this procedure?" Twenty patients (66\%) responded that the effects of this procedure persisted for longer than four months.

Question 2: Do you think how long exist the effect of this procedure?

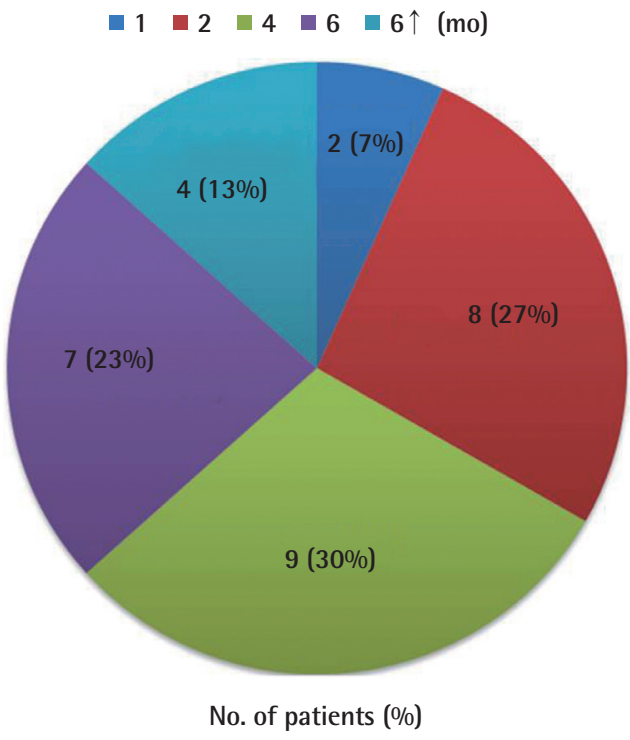

Table 1. Clinical scoring of five subjects

\begin{tabular}{|lcc|}
\hline Variable & Mean (standard deviation) & P-value \\
\hline Skin surface roughness & $3.67(0.50)$ & $0.000^{\text {a) }}$ \\
Fine wrinkles & $3.43(0.52)$ & $0.000^{a)}$ \\
Moisture & $3.42(0.56)$ & $0.000^{\text {a) }}$ \\
Brightness & $3.78(0.49)$ & $0.00^{\text {a) }}$ \\
Elasticity & $3.60(0.51)$ & $0.000^{a)}$ \\
\hline
\end{tabular}

Evaluation by three plastic surgeons showed statistically significant changes in improvement of skin surface roughness, fine wrinkles, moisture, brightness and elasticity $(P<0.05)$.

a)Statistically significant with $\mathrm{P}<0.05$.

Table 2. Comparison of clinical scoring of 3 plastic surgeons

\begin{tabular}{|c|c|c|c|c|}
\hline \multirow{2}{*}{ Variable } & \multicolumn{3}{|c|}{ Score of three doctors, mean (standard deviation) } & \multirow{2}{*}{ P-value } \\
\hline & $1(n=30)$ & $2(n=30)$ & $3(n=30)$ & \\
\hline Skin surface roughness & $3.633(0.556)$ & $3.733(0.450)$ & $3.633(0.490)$ & $0.672^{\mathrm{a})}$ \\
\hline Fine wrinkles & $3.400(0.498)$ & $3.467(0.507)$ & $3.433(0.568)$ & $0.886^{a)}$ \\
\hline Moisture & $3.400(0.563)$ & $3.533(0.571)$ & $3.333(0.547)$ & $0.990^{\mathrm{a})}$ \\
\hline Brightness & $3.733(0.450)$ & $3.767(0.568)$ & $3.833(0.461)$ & $0.316^{\mathrm{a})}$ \\
\hline Elasticity & $3.533(0.507)$ & $3.667(0.547)$ & $3.600(0.498)$ & $0.479^{\mathrm{a})}$ \\
\hline
\end{tabular}


in three sessions at intervals of four weeks. We found that she showed improvement of brightness, skin surface roughness and elasticity ( 4 point, 4 point, 4 point; average value). In addition, she was satisfied with the results of this treatment in improvement of skin surface roughness and brightness (4 point; satisfied) (Fig. 3)

Case 2: a 60-year-old female patient was injected at whole face in three sessions at intervals of four weeks. We also found that she showed improvement of brightness, skin surface roughness and elasticity ( 4.33 point, 4 point, 4 point; average value), and she was satisfied with the results of this treatment in improvement of skin surface, brightness and fine wrinkles (4 point; satisfied) (Fig. 4).

\section{DISCUSSION}

Concentration of HA in skin decreases with aging. As a result, ability to hold water and elasticity also decrease. Therefore, volume in skin decreases, and tendency of wrinkle formation in skin shows a gradual increase. In addition, there are other major extrinsic factors that affect skin aging [1-3]. Approximately $80 \%$ of facial skin aging occurs through ultraviolet (UV)-exposure. Initially, UV radiation causes damage to skin, and induces a wound healing response, leading to an increase in dermal HA. However, repeated and extensive exposure to UV radiation ultimately causes a decrease in dermal HA and typical wound healing response is stimulated, resulting in formation of scar-like tissue, which has low pliability $[1,4]$. Thus, many people are interested in procedures for rejuvenation of the aged appearance. Because HA is identical chemically and molecularly in tissue of all mammals, there is species-nonspecificity [5]. In 2003, Restylane gel (Q-

Table 4. Objective scoring of moisture, oil and elasticity

\begin{tabular}{|c|c|c|}
\hline Variable & Mean (standard deviation) & P-value \\
\hline Moisture difference & $10.00(1.33)$ & $0.000^{\mathrm{a})}$ \\
\hline Oil difference & $-4.20(1.55)$ & $0.000^{\mathrm{a})}$ \\
\hline Elasticity difference & $11.90(1.66)$ & $0.000^{\mathrm{a})}$ \\
\hline \multicolumn{3}{|c|}{$\begin{array}{l}\text { Evaluation showed statistically significant changes in moisture, oil and elasticit } \\
\text { improvement in ten patients injected HA filler }(P<0.05) \text {. } \\
\text { a) Statistically significant with } P<0.05 \text {. }\end{array}$} \\
\hline
\end{tabular}

\section{Table 3 . Comparison of 5 subjective evaluated by 3 plastic surgeons}

\begin{tabular}{|c|c|c|c|c|c|c|}
\hline \multirow{2}{*}{ Degree of satisfaction } & \multicolumn{5}{|c|}{ Satisfaction of 5 factor } & \multirow{2}{*}{ P-value } \\
\hline & Skin surface roughness ${ }^{1}$ & Fine wrinkles ${ }^{2}$ & Moisture $^{3}$ & Brightness $^{4}$ & Elasticity $^{5}$ & \\
\hline Mean (standard deviation) & $3.67(0.50)$ & $3.43(0.52)$ & $3.42(0.56)$ & $3.78(0.49)$ & $3.60(0.51)$ & $\begin{array}{c}0.000^{\mathrm{a})} \\
2,3<1,4,5^{\text {b) }}\end{array}$ \\
\hline
\end{tabular}

Fig. 3. Case 1: 35-year-old female patient

(A) Photograph before treatment.

(B) Photograph at 6 months after last treatment session.
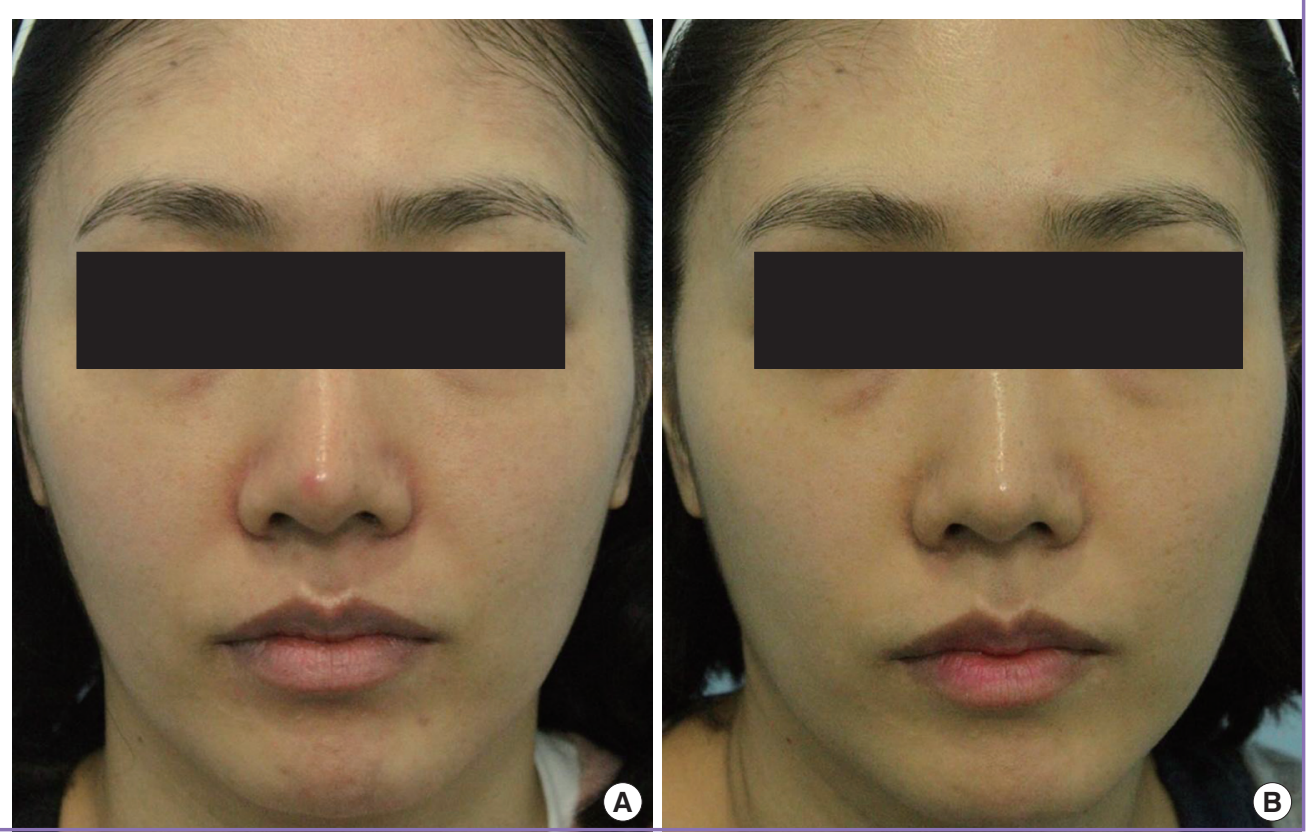


\section{Fig. 4. Case 2: 60-year-old female patient}

(A) Photograph before treatment.

(B) Photograph at 6 months after last treatment session.
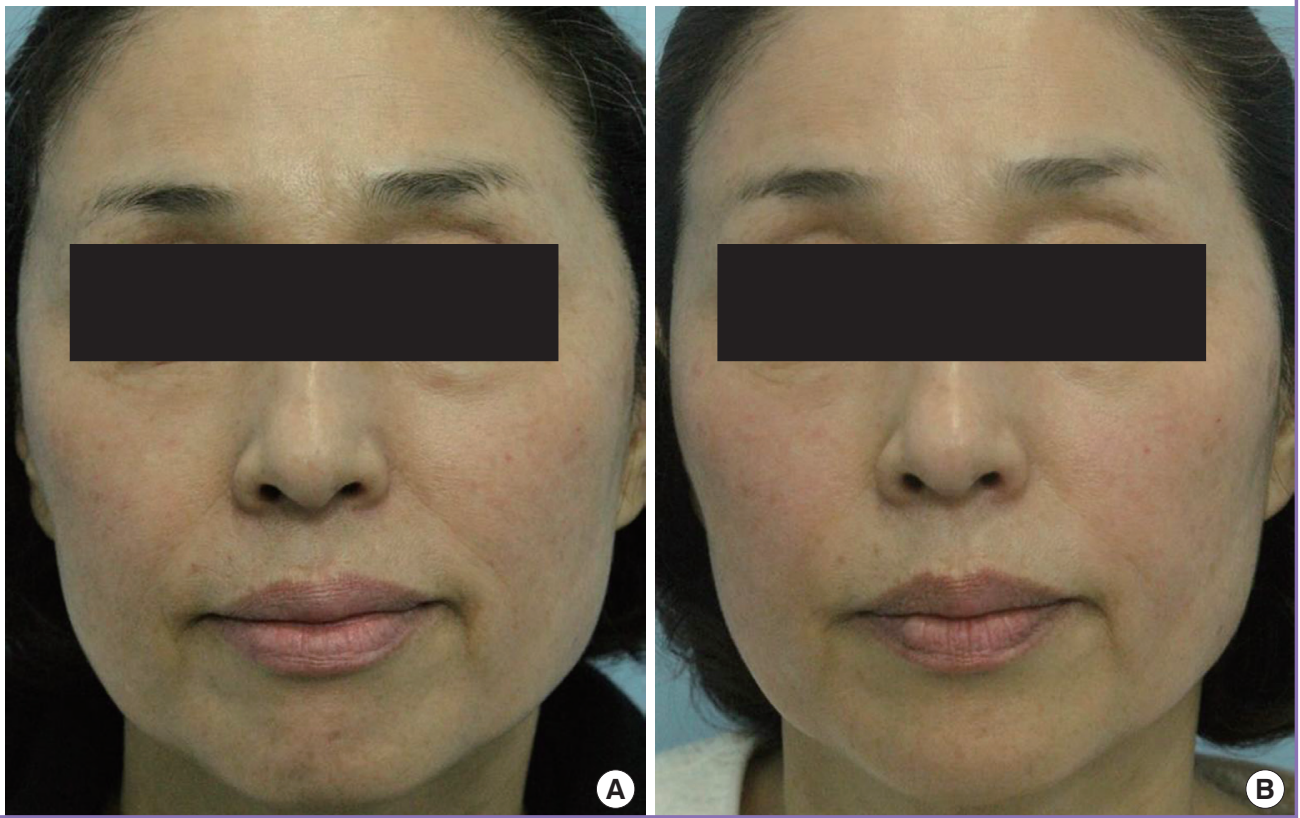

Fig. 5. Schematic effect of hyaluronic acid in skin

(A) Dry and tired skin. (B) Hyaluronic acid gels are added, acting as a dermal reservoir of hydration. (C) Hydrobalance of skin is restored. Hydrated and healthy skin results in improvement of skin surface roughness and facial wrinkles.
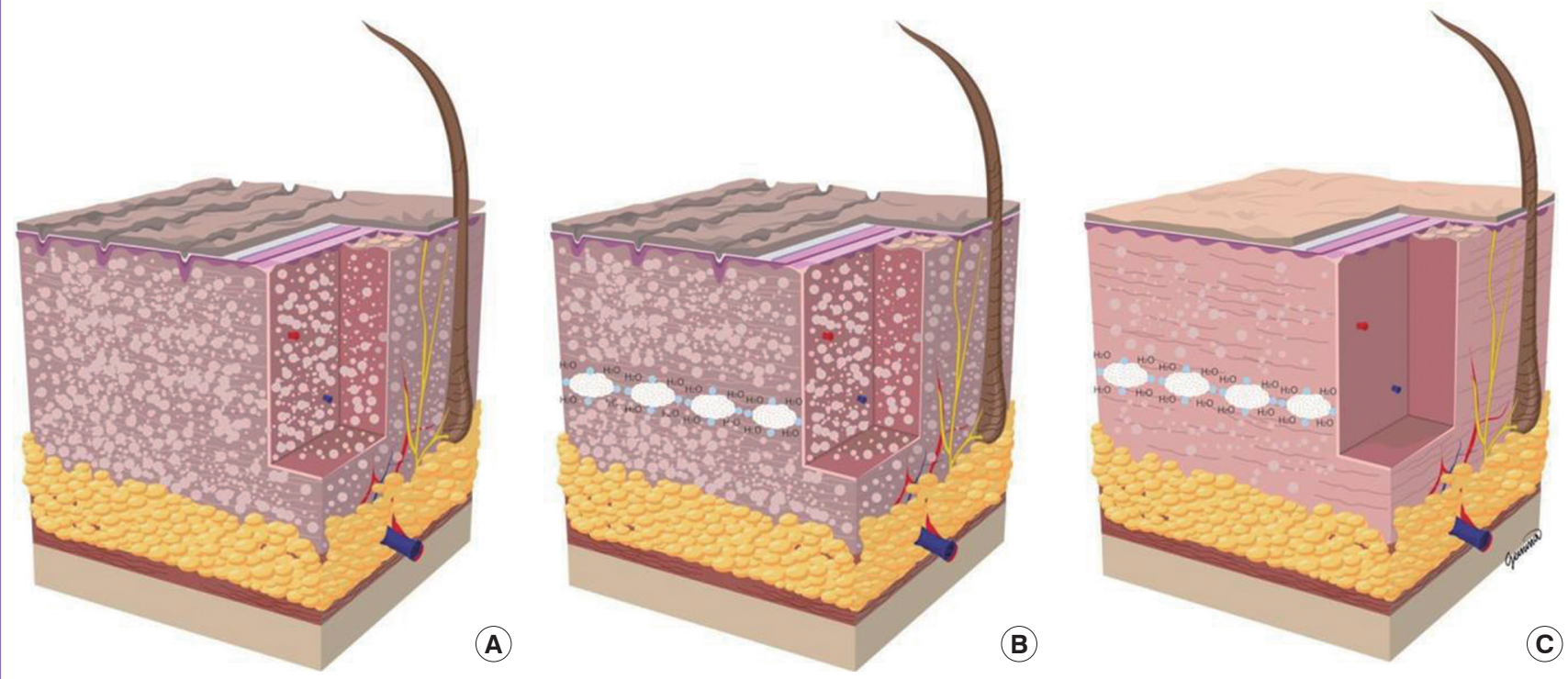

Med, Uppsala, Sweden) was approved by the Food and Drug Administration for treatment of moderate to severe facial wrinkles and it is used in more than 60 countries [6]. Since its approval, HA has accounted for $85 \%$ of whole filler for facial rejuvenation $[5,7]$. Approval of these HA products is a significant development in facial aesthetic procedures.

HA filler has been used for improvement of deep wrinkle and facial contour. It is one of the most popular procedures all over the world. This procedure is performed through injection of a certain amount of $\mathrm{HA}$ filler into the subdermal layer.

Recently, by injection of HA filler into the intradermal layers, not subdermal layers, in which shows better volume effects, there has been interest in skin rejuvenation. Intradermal injections of HA play a role as a dermal reservoir of hydration, by drawing water into extracellular matrix in dermis, resulting in hydration in dermis and improvement of skin surface roughness and fine wrinkles (Fig. 5) [8].

A recent study reported that intradermal injection of NASHA 
(Restylane Vital, Q-Med, Uppsala, Sweden) can promote collagen production. Compared to the control group, accumulation of collagen was observed in the area of the surrounding filler. Another study demonstrated that injection of small quantities of NASHA over large areas of the dermis resulted in improved elasticity and rough skin surface $[9,10]$. We study the clinical effects of intradermal injection of $\mathrm{HA}$, which has been reported in very few cases.

To evaluate the effects of the procedure, we asked patients to complete a questionnaire consisting of four questions after 6 months of last procedure. In addition, three plastic surgeons selected five subjects for evaluation of improvement of skin texture (improvement of skin surface roughness, fine wrinkles, moisture, brightness and elasticity), and the average value of outcomes was then calculated.

In this study, the majority of patients (77\%) were satisfied with the therapeutic outcomes. Approximately $66 \%$ of patients responded that the effects of this procedure persisted for longer than four months, and the majority of patients (77\%) wanted to undergo this procedure again and would recommend this procedure to acquaintances. Regarding doctor's evaluation, we found that improvement of brightness showed the highest score for satisfaction. And scores for improvement of brightness, skin surface roughness and elasticity was significantly higher than those for improvement of fine wrinkle and moisture.

For more objective evaluation, moisture, oil, and elasticity were measured before the procedure and before the last treatment session in 10 patients. Results of the evaluations showed statistically significant changes in moisture, oil, and elasticity improvement $(\mathrm{P}<0.05)$. Elasticity showed the most improvement, followed by moisture and oil.

In this trial, we used an automatic injector for precise injection of HA filler into the intradermal layer. AQUAGENT is an injection system that shortened down time by simultaneous use of the needling stamp system and multi-pin medical liquid injection. This system can be used for control of even tiny layers of skin by precise needling and high speed injection. Few side effects have been reported. We can use a freely available needling type (one-pin needle, five-pin needle, catheter needle). A vertical or horizontal procedure is also available in accordance with facial shape and curve. It can be used in the obesity clinic, pigmentation clinic, wrinkle clinic, and scalp clinic. Using this system, no serious adverse events occurred during the course of treatment.

Most side effects of intradermal injections of Restylane Vital are mild and transient and include pain and intermittent swelling, edema, erythema, and ecchymosis at the injection site [6]. Technique-related side effect is from irregular facial contour and lasting beading caused by superficial injection of filler to skin necrosis caused by vascular occlusion [5]. Foreign body granuloma caused by HA injection has been reported in another study [11].

No serious adverse events occurred during the course of treatment, and none of the patients withdrew from the study due to adverse effects. Three patients experienced temporary, mild erythema after treatment (lasting approximately one week). Two patients developed transient, mild hematomas after treatment.

Findings of this study demonstrate that intradermal injection of Restylane Vital is a useful rejuvenation procedure, which can contribute to happiness and confidence of patients. However, conduct of larger group studies will be needed for further evaluation of safety, effectiveness, and reliability of this procedure.

\section{REFERENCES}

1. Uitto J. Understanding premature skin aging. N Engl J Med 1997;337:1463-5.

2. Stern R, Maibach HI. Hyaluronan in skin: aspects of aging and its pharmacologic modulation. Clin Dermatol 2008;26: 106-22.

3. Meyer LJ, Stern R. Age-dependent changes of hyaluronan in human skin. J Invest Dermatol 1994;102:385-9.

4. Carruthers A, Carruthers J. Non-animal-based hyaluronic acid fillers: scientific and technical considerations. Plast Reconstr Surg 2007;120:33S-40S.

5. Matarasso SL. Understanding and using hyaluronic acid. Aesthet Surg J 2004;24:361-4.

6. Jordan DR. Soft-tissue fillers for wrinkles, folds and volume augmentation. Can J Ophthalmol 2003;38:285-8.

7. Jang JC, Shin SH, Han SK, et al. The efficacy of new hyaluronic acid filler (HyaFilia). J Korean Soc Plast Reconstr Surg 2011;38:1-6.

8. Matarasso SL, Carruthers JD, Jewell ML, et al. Consensus recommendations for soft-tissue augmentation with nonanimal stabilized hyaluronic acid (Restylane). Plast Reconstr Surg 2006;117:3S-34S.

9. Wang F, Garza LA, Kang S, et al. In vivo stimulation of de novo collagen production caused by cross-linked hyaluronic acid dermal filler injections in photodamaged human skin. Arch Dermatol 2007; 143:155-63.

10. Kerscher M, Bayrhammer J, Reuther T. Rejuvenating influence of a stabilized hyaluronic acid-based gel of nonanimal origin on facial skin aging. Dermatol Surg 2008;34:720-6.

11. Yang JH, Lee SM, Won $\mathrm{CH}$, et al. Foreign body granuloma caused by hyaluronic acid/dextranomer microsphere filler injection. Int J Dermatol 2012;51:1517-8. 